\title{
Emerging Technologies for Computing
}

\author{
Giovanni De Micheli
}

\section{EPFL}

The evolution of computing has lead to very different realizations to cope with performance and energy consumption density, two competing factors that are not obvious to reconcile. The current semiconductor technologies, mainly FinFETs and FDSoI, provide us with a robust physical means of addressing computing requirements, but a strong interest is placed now in how to enhance the current devices with new materials as well as how to use these materials to realize completely novel devices. Medium-term enhancement of silicon technology includes tunnel FETs and NanoWire (NW) transistors, which both can address low-power solutions. Recent research has also shown the usefulness of Carbon NanoTubes (CNTs) in realizing simple but scalable computational engines, as well as 2Dimensional materials, such and $\mathrm{MoS}_{2}$ to realize transistors. A different approach to increasing the computational effectiveness of transistors is electrostatic doping, i.e., the realization of transistors that can have electrically-programmable polarity. Such devices have been realized on various substrates, such as $\mathrm{SiNW}, \mathrm{CNTs}$ and $\mathrm{WSe}_{2}$ in experimental forms. Moreover, memory technology provides us with the capability of realizing large arrays, a part of which can be dedicated to computing, namely inmemory computing. In the specific case or Resistive RAMs, each cell has a sequential behavior that can be unrolled to provide logic computation. Design algorithms, methods and tools, such as those based on majority algebra, are both important investigation and design means for CMOS-based, controlled-polarity gates and ReRAM in-memory circuits. Eventually, the evolution of semiconductor technology will bring us the fusion of sensors with computation, thus enabling new computing modalities and interaction with the physical environment. Broadly speaking, geometry downscaling, material hybridization and multi-layer integration are the ingredients for more diverse and powerful computing paradigms in the years to come.

Giovanni De Micheli is Professor and Director of the Institute of Electrical Engineering at EPFL Lausanne, Switzerland. He is program leader of the Nano-Tera.ch program. Previously, he was Professor of Electrical Engineering at Stanford University. He is a Fellow of ACM and IEEE, member of the Academia Europaea and Foreign Honorary Member of the American Academy of Arts and Sciences.

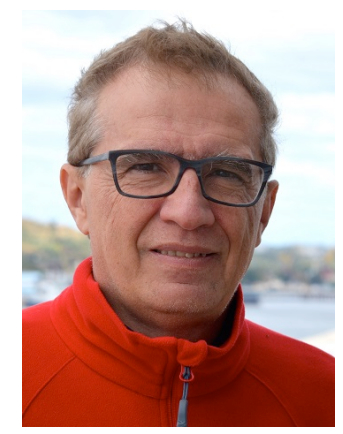

